\title{
A germline variant of human IgG1+ B cell receptor represses colorectal tumorigenesis and progression by shaping the tumor microenvironment
}

\author{
Wanli Liu ( $\square$ liulab@tsinghua.edu.cn ) \\ Tsinghua University https://orcid.org/0000-0003-0395-2800 \\ Bing Yang \\ Tsinghua University \\ Zheng Zhang \\ Peking University People's Hospital \\ Xiangjun Chen \\ Tsinghua University \\ Xu-Yan Wang
}

Sun Yat-sen University

Shishang Qin

Peking University

Liaoqi Du

Tsinghua University

Changjiang Yang

Peking University People's Hospital

Wenbo Sun

Tsinghua University

Liyu Zhu

Peking University People's Hospital

Qinwen Zheng

Fudan University

Yongjie Zhu

Tsinghua University

Xiya Song

Tsinghua University

Shidong Zhao

Peking University People's Hospital

Quan Wang

Peking University People's Hospital 


\section{Long Zhao}

Peking University People's Hospital

\section{Yilin Lin}

Peking University People's Hospital

\section{Xiaohan Yan}

Tsinghua University

\section{Ji Gao}

Tsinghua University

\section{Jinghe Huang}

Shanghai Public Health Clinical Center and Key Laboratory of Medical Molecular Virology (MOE/NHC/CAMS), School of Basic Medical Sciences, Fudan University, 201508 https://orcid.org/00000002-1968-871X

\section{Fan Wu}

Shanghai Public Health Clinical Center and Key Laboratory of Medical Molecular Virology (MOE/NHC/CAMS), School of Basic Medical Sciences, Fudan University, 201508

\section{Lu Lu}

Key Laboratory of Medical Molecular Virology (MOE/NHC/CAMS), School of Basic Medical Sciences, Fudan University, 130 Dong An Rd., Shanghai 200032 https://orcid.org/0000-0002-2255-0391

\section{Fei Wang}

Chinese Academy of Sciences

\section{Wenjie Zheng}

Peking Union Medical College Hospital

\section{Xiao-Hua Zhou}

Peking University

\section{Xiaozhen Zhao}

Peking University People's Hospital

\section{Ziye Wang}

Peking University People's Hospital

\section{Xiaolin Sun}

Peking University People's Hospital https://orcid.org/0000-0001-9709-1532

\section{Yingjiang Ye}

Peking University People's Hospital

\section{Shan Wang}

Peking University People's Hospital

\section{Zhan-Guo Li}

People's Hospital, Peking University Health Science Centre https://orcid.org/0000-0002-2590-6242

\section{Hai Qi}

School of Medicine, Tsinghua University https://orcid.org/0000-0001-5475-3989

\section{Zemin Zhang}


Peking University https://orcid.org/0000-0003-3789-6536

\section{Dong-Ming Kuang}

Sun Yat-sen University https://orcid.org/0000-0003-3570-9717

\section{Lei Zhang}

Peking University

\section{Zhanlong Shen}

Peking University People's Hospital

\section{Research Article}

Keywords: tumor tertiary lymphoid structures (TLSs), gastrointestinal cancer, colorectal cancer

Posted Date: January 27th, 2021

DOI: https://doi.org/10.21203/rs.3.rs-151780/v1

License: (c) (1) This work is licensed under a Creative Commons Attribution 4.0 International License. Read Full License 


\section{Abstract}

The presence of B lymphocytes in tumor tertiary lymphoid structures (TLSs) is an important prognostic indicator for different types of cancers. However, whether B cell responses in the tumor microenvironment (TME) can be harnessed for immunotherapy is unclear. Here we report that a protective germline variant of human immunoglobulin heavy constant gamma 1 gene (IGHG1) containing a Gly396 to Arg396 substitution (hlgG1-G396R) confers improved survival of colorectal cancer (CRC) patients. These hlgG1G396R homozygous CRC patients displayed elevated tumor-associated antigen (TAA)-specific IgG1 antibody production and plasma cell infiltration into tumors. In murine colon carcinoma models, mice expressing the murine functional homolog IgG2c-Gly400Arg variant (mlgG2c-G400R) also produce higher levels of tumor-specific IgG2c antibodies via enhanced plasma cell differentiation, together with alleviated tumorigenesis and progression. Mechanistically, this variant potentiates TAA-specific antibodydependent cellular phagocytosis and antigen presentation. Comprehensive immune profiling of the TME of CRC patients revealed that hlgG1-G396R prominently promotes broad mobilization of immune cells ( $\operatorname{lgG} 1^{+}$plasma cells, $\mathrm{CD} 8^{+} \mathrm{T}$ cells, $\mathrm{CD} 103^{+} \mathrm{DCs}$ ) and efficient TLS formation, both key components of an anti-tumor microenvironment. Notably, adoptive transfer of tumor-primed B cells with this variant exhibited therapeutic efficacy in murine tumor models, demonstrating clinical potential. These results prompt a prospective investigation of hlgG1-G396R in CRC patients as a biomarker for clinical prognosis and demonstrate that manipulating the functionality of $\lg \mathrm{G}^{+} \mathrm{B}$ cells in tumors could improve immunotherapy outcomes.

\section{Main Text}

Tumor cells evade clearance by the immune system via several mechanisms, including inhibiting neoantigen presentation and inducing immune silencing in the tumor microenvironment (TME). Extensive studies have focused on effective ways to reinvigorate immunosurveillance by modulating $T$ lymphocyte activation through $T$ cell receptor (TCR) engineering or immune checkpoint blockade ${ }^{1}$. However, the response rate of immunotherapy is still low based on current paradigms ${ }^{2-4}$. Although growing numbers of studies suggest credible roles also for $B$ lymphocytes in anti-tumor immunity ${ }^{5-8}$, it is unclear whether manipulation of $B$ cell antigen receptor (BCR) activation could facilitate immunosurveillance against tumors.

Within the TME, tumor-associated antigen (TAA) can drive tumor-specific $B$ cells to undergo immune activation, class-switch to $\lg \mathrm{G}^{+}$memory $\mathrm{B}$ cells and differentiation to $\lg \mathrm{G} 1$ antibody-secreting plasma cells ${ }^{9}$. A single nucleotide polymorphism (SNP) rs117518546 (C > T in allele, Glycine > Arginine in amino acid, hlgG1-G396R) located in human immunoglobulin heavy constant gamma 1 gene (IGHG1) was recently reported to enhance the activation of IgG1-BCR signaling and promote $\lg$ 1 $^{+}$plasma cell polarization ${ }^{10}$. Here, we investigated whether this variant could also modulate cancer progression and survival by focusing on a potential role in colorectal cancer (CRC). 
To determine whether the presence of the hlgG1-G396R variant is correlated with clinical colorectal tumorigenesis, we established a cohort containing 1006 CRC patients with up to 100-month survival clinical records from Peking University People's Hospital in Beijing China (Extended Data Fig. 1, Table 1). The frequency of the hlgG1-G396R homozygotes was decreased in the CRC patients compared to criteriamatched controls ( $1006 \mathrm{CRC}$ patients versus 583 healthy controls, odds ratio $=0.747, P=0.049$; Extended Data Table 2), suggesting a potential protective role of this variant in CRC disease. For further validation, we compared the cumulative overall survival (OS) of 537 wild-type (WT) patients, 376 hlgG1-G396R heterozygous patients and 93 hlgG1-G396R homozygous CRC patients, and found that hlgG1-G396R homozygous patients exhibited substantially improved OS than WT patients (Log-rank $P=0.007$; Fig. 1A). A standard and comprehensive epidemiological comparison indicated that the proportion of 100-month cumulative OS was $78.16 \%(95 \% \mathrm{Cl}, 68.59 \%-89.06 \%)$ for hlgG1-G396R homozygous patients, compared with $59.34 \%$ (54.29\%-64.85\%) for WT patients and 58.79\% (52.61\%-65.68\%) for hlgG1-G396R heterozygous patients (Log-rank $P=0.028$; Fig. 1B). In addition to these OS clinical analyses, the hlgG1G396R variant was also significantly associated with superior progression-free survival (PFS) in a large proportion of CRC patients $(n=966)$, which had obtainable follow-up PFS clinical information from the original cohort of 1006 patients. Consistent with the OS analysis, hlgG1-G396R-mediated benefit to PFS was more striking when comparing hlgG1-G396R homozygotes to WT CRC patients (Log-rank $P=0.018$; Extended Data Fig. 2A). The proportion of 100 -month cumulative PFS was $75.75 \%(95 \% \mathrm{Cl}$, 66.51\%-86.27\%) for hlgG1-G396R homozygous patients, compared with 58.47\% (52.41\%-65.24\%) for hlgG1-G396R heterozygous patients and 57.87\% (52.92\%-63.29\%) for WT patients (Log-rank $P=0.052$; Extended Data Fig. 2B). Notably, multivariable COX regression analyses implicated the hlgG1-G396R variant as an independent positive prognostic factor for both OS (Fig. 1C, Extended Data Table 3) and PFS (Extended Data Fig. 2C, Extended Data Table 3). Furthermore, hlgG1-G396R was consistently identified as a protective factor in PFS (Extended Data Fig. 2D), OS (Extended Data Fig. 3) and allele frequency (Extended Data Table 4) based stratification analyses. Thus, hlgG1-G396R is a newly defined protective germline variant for progression and survival in CRC patients.

To determine the mechanism of action of hlgG1-G396R in inhibiting CRC tumorigenesis, we generated knock-in mice harboring the homologous mutation at the cytoplasmic tail of endogenous murine lgG2c (denoted as mlgG2c-G400R) in consideration of murine lgG2c sharing a conserved tail sequence and functional equivalence to human $\lg \mathrm{G}^{11}$ (Extended Data Fig. 4A, B). In a transplantation tumor model using syngeneic MC38 murine colon carcinoma cells, mlgG2c-G400R mice showed significantly slower progression of inoculated MC38 tumor cells compared to the WT control mice (Fig. 1D, E). In order to more closely simulate colon tumorigenesis in humans, we introduced the azoxymethane (AOM) / dextran sodium sulfate (DSS) colitis-associated carcinoma (CAC) model to our study, which has been widely used for the induction of colitis-dependent neoplasia and primary cancer in mice (Extended Data Fig. 4C) ${ }^{12,13}$. In mlgG2c-G400R mice, the AOM-DSS treatment reduced weight loss (Fig. 1F, Extended Data Fig. 4D), decreased the disease activity index (Fig. 1G), alleviated phenotypes of tumor-associated inflammation (Fig. 1H, Extended Data Fig. 4E, F), reduced tumor burden (Fig. 1I, Extended Data Fig. 4G), and ameliorated histopathological changes (Fig. 1J, Extended Data Fig. 4H) compared to the WT control 
mice. Thus, the mlgG2c-G400R variant alleviates tumorigenesis and tumor progression in mice, recapitulating the results of the functional equivalent hlgG1-G396R variant in human CRC patients.

Given that the hlgG1-G396R variant enhances $\operatorname{lgG} 1^{+} \mathrm{B}$ cells activation and $\lg \mathrm{G} 1$ producing plasma cell differentiation ${ }^{10}$, and that human IgG1 antibodies are important modulators in immunosurveillance ${ }^{14,15}$, we investigated whether the hlgG1-G396R variant differentially regulates the production of tumor-specific IgG1 antibodies. In the MC38 transplantation model, which presents a broad TAA pool ${ }^{16}$, mlgG2c-G400R mice generated elevated amounts of MC38 cell-specific IgG2C antibodies compared to WT mice (Fig. 2A). Tumor-primed $B$ cells isolated from the tumor-draining lymph nodes (TDLNs) of mice could secret large amounts of IgG under the in vitro re-stimulation with tumor cells, comparing with unprimed $B$ cells (Extended Data Fig. 5A) ${ }^{17}$. B cells from the TDLNs of mlgG2c-G400R mice secreted significantly higher amounts of IgG2C upon re-stimulation with irradiated MC38 cells than B cells from TDLNs of WT mice (Fig. 2B). Consistently, the levels of intestinal IgG2C antibodies were significantly increased in mlgG2CG400R mice upon AOM/DSS induction, while the levels of intestinal IgA, IgG1, IgG2b and IgG3 antibodies in mlgG2c-G400R mice were comparable with WT mice (Fig. 2C).

To comprehensively characterize the TAA-specific antibodies, we used microarrays to profile tumor specific antibody levels against a panel of 51 TAAs. The results further validated the burst of sera IgG2c antibody production with broad TAA specificity, but not sera IgM, IgG1 or IgG2b antibodies in mlgG2cG400R CAC mice (Fig. 2D, Extended Data Fig. 5B). Similarly, in CRC patients, the hlgG1-G396R homozygotes produced elevated levels of TAA-specific IgG1 antibodies (Fig. 2E), such as anti-AFP, antiCEA and anti-CA125 antibodies (Fig. 2F), while this stimulatory effect was not significant for IgM antibodies. Previous studies have indicated human IgG3 as a negative prognostic marker for cancer patients, which is the opposite of human $\lg 1^{18,19}$. Notably, we identified an increase in the ratio of $\operatorname{lgG} 1$ to IgG3 TAA-specific antibodies in hlgG1-G396R homozygous patients (Extended Data Fig. 5C, D). The boosted production of TAA-specific IgG2C antibody was also detected in the colon explants of CACinduced mice by TAA microarrays (Extended Data Fig. 5E). Together, these results demonstrate that the hlgG1-G396R variant boosts the production of TAA-specific IgG1 antibodies in human CRC patients, similar to the mlgG2c-G400R variant in tumor-bearing mice.

To investigate the role of this variant in immunosurveillance mediated by TAA-specific IgG1 antibody upregulation, we utilized membrane-bound ovalbumin (OVA)-expressing MC38 tumor cells (denoted as MC38-mOVA) and B16F10-mOVA (denoted as B16-mOVA) (Extended Data Fig. 5F) to examine the effect of OVA priming on anti-tumor immunity in the mlgG2c-G400R mice. To establish a loss-of-function control in our experimental system, we constructed a new mlgG2c-tailless mouse with a truncated cytoplasmic tail of the membrane-bound mlgG2c heavy chain, which blocks the mlgG-tail signaling transduction for enhanced $\operatorname{lgG}^{+} B$ cell activation and differentiation ${ }^{20,21}$. After immunization of OVA antigen combined with Th1-prone adjuvant ${ }^{22}$, which favors IgG2c antibody production, OVA-specific IgG2c antibody production significantly increased in mlgG2c-G400R mice while decreasing in mlgG2ctailless mice compared to WT mice (Fig. 2G). Notably, tumor growth was clearly inhibited in mlgG2C- 
G400R mice during the subsequent MC38-mOVA challenge, whereas it was exacerbated in mlgG2ctailless mice compared with WT mice (Fig. 2H, Extended Data Fig. 5G). Consistently, in the B16-mOVA tumor cell inoculation model, mlgG2c-G400R mice exhibited slower tumor progression and prolonged survival compared to WT mice, while the benefits were diminished in mlgG2c-tailless mice (Fig. $2 \mathrm{H}$ ). Further linear regression fitting analyses indicated that OVA-specific IgG2c antibody levels negatively correlated with MC38-mOVA tumor size and positively correlated with survival time in the B16-mOVA transplantation tumor model (Fig. 2I).

Mechanistically, we detected higher percentages of OVA-specific IgG2 $\mathrm{c}^{+}$germinal center (GC) B cells and memory B cells, as well as an increase of plasma cells in the spleen of mlgG2c-G400R mice compared to WT mice after OVA priming (Fig. 2J, Extended Data Fig. 5H). Moreover, the substantial increase of OVAspecific lgG2 $\mathrm{c}^{+}$plasma cells and memory $B$ cells in mlgG2c-G400R mice were also detected in the bone marrow (Fig. 2K). Similarly, higher percentages of class switched IgG2 $\mathrm{c}^{+}$plasma cells and memory $\mathrm{B}$ cells were observed in the murine mesenteric $L N s$ ( $m L N s)$, the lamina propria (LP) of AOM/DSS induced mlgG2c-G400R CAC mice (Extended Data Fig. 6A), and the TDLNs of MC38 tumor bearing mlgG2c-G400R mice (Extended Data Fig. 6B). More importantly, elevated infiltration of plasma cells (Fig. 2L) and IgG1 (Fig. 2M, N) in tumor sections from hlgG1-G396R homozygous CRC patients were consistently detected by IHC staining, while there were comparable numbers of total B cells. Specifically, we detected higher levels of immunoglobulin heavy constant gamma $1\left(C_{Y} 1\right)$ transcripts in the tumor tissues from hlgG1G396R homozygous CRC patients, which is a hallmark of elevated IgG1 expression in the $\mathrm{TME}^{23}$, while the levels of $C_{\gamma 2}, C_{\gamma} 3, C_{\gamma} 4$ and immunoglobulin heavy constant alpha $(C a)$ transcripts were comparative (Fig. 20). Thus, mlgG2c-G400R alleviates mice from colorectal tumor progression by promoting TAAspecific $\lg G 2$ c producing plasma cell differentiation and subsequent IgG2C antibody production within the TME.

The interactions between immune system and tumor are governed by a complex network of cell-cell interactions. Thus, we considered that the hlgG1-G396R variant may exert a pleiotropic immunomodulatory role by also affecting interrelated immune cells for effective immunosurveillance. To test this, we analyzed the effect of the variant on infiltration of both innate and adaptive immune cells in the TME, including T cells, natural killer (NK) cells, macrophages and DCs. In the MC38-mOVA tumor model, mlgG2c-G400R mice exhibited increased amounts of $\mathrm{CD} 8^{+} \mathrm{T}$ cells within subcutaneous tumor tissues (Fig. 3A), and especially increased $C D 44^{\text {high }} \mathrm{CD} 62 \mathrm{~L}^{\text {low }} \mathrm{CD} 8^{+}$effector $\mathrm{T}$ cells, with elevated levels of cytolytic granzyme B and interferon-y (IFN- $\gamma$ ) production (Fig. 3B, C). This indicates that tumorinfiltrating T cells from MC38-inoculated mlgG2C-G400R mice maintain an optimal functionality, which may help to limit tumor progression. Notably, DCs, especially $\mathrm{CD} 103^{+} \mathrm{CDC1}$, which are the key antigen presenting cells transporting antigens to lymphoid structure and priming tumor-specific $\mathrm{CD} 8^{+} \mathrm{T}$ cells ${ }^{24}$, were also elevated in mlgG2c-G400R mice compared to WT mice (Fig. 3D). The MHC-I presentation of OVA epitopes by $\mathrm{CD}_{103^{+}} \mathrm{CDC} 1$ had a moderate increase in the tumor tissues from mlgG2c-G400R mice (Fig. 3E). The amounts of tumor-infiltrating macrophages, NK cells and NKT cells displayed no significant differences (Extended Data Fig. 6C). Remarkably, IHC staining revealed that the colon tumor specimens 
from CAC-induced mlgG2c-G400R mice exhibited dramatically increased infiltration specifically of CD8 ${ }^{+} \mathrm{T}$ cells compared with those from WT mice (Fig. 3F, Extended Data Fig. 7A, B).

Tertiary lymphoid structures (TLSs) were recently identified as important indicators of favorable outcomes of immunotherapies $^{5-7}$. B cells and DCs have been shown to be the major initiators of TLS formation, and together with T cells are key constituents of $\mathrm{TLS}^{25}$. In addition, plasma cells and antibodies are positively associated with TLS formation. IHC staining revealed a trend of more and larger TLSs formation within the colon tumors of mlgG2c-G400R mice (Fig. 3G). The elevated immune cell infiltration and TLS formation were largely recapitulated in the tumor tissues of CRC patients: hlgG1G396R homozygotes had prominently increased tumor infiltrating $\mathrm{CD}^{+} \mathrm{T}$ cells accompanied with an accumulation of $\mathrm{S} 100^{+}$DCs compared with other genotypes (Fig. 3H, Extended Data Fig. 7C, D). There was also a mild but consistent increase of TLSs in the tumor specimens from hlgG1-G396R homozygotes in comparison with WT (Fig. 3I). The transcriptional levels of five chemokines (CXCR5, CXCL12, CXCL13, CCL19, CCL21) that have been reported to be involved in the formation of TLS ${ }^{26}$, were prominently elevated in both the tumor specimens from mlgG2c-G400R mice and hlgG1-G396R homozygous CRC patients (Fig. 3J). Collectively, these findings suggest that the hlgG1-G396R variant potentiates anti-tumor responses also by invigorating $\mathrm{CD} 8^{+}$effector $\mathrm{T}$ cell priming and activation by $\mathrm{DCs}$ within TLSs.

Single cell RNA sequencing (scRNA-seq) is widely used to profile immune cells in organs and tissues. Thus, we re-analyzed the proportions of immune cell subsets in the TME of $18 \mathrm{CRC}$ patients using the scRNA-seq results from our recent studies ${ }^{27,28}$. Both patient genome sequencing and single B cell RNAseq data identified 11 heterozygotes, 6 WT and only one hlgG1-G396R homozygote within these 18 CRC patients, so we could only compare immune cell profiles within the TME from 12 hlgG1-G396R carriers $(\mathrm{CT}+\mathrm{TT}$ ) to those from 6 non-carriers (CC) (Extended Data Table 5). Nevertheless, in hlgG1-G396R carriers, there were increased proportions of $I g G^{+}$plasma cells, $C X C R 5^{+} \mathrm{T}$ follicular helper cells, and $C D 6^{+}$ tumor-resident memory T cells, and decreased proportions of $C T L A 4^{+}$regulatory T cells (Fig. $3 \mathrm{~K}, \mathrm{~L}$, Extended Data Fig. 7E). Moreover, the proportions of $L A Y N^{+}$exhausted T cells were also decreased in hlgG1-G396R carriers, implying that the variant may be beneficial to ameliorate $T$ cell exhaustion (Fig. 3L). All these results indicate that the hlgG1-G396R variant favors the formation of an anti-tumor microenvironment.

Human $\lg \mathrm{G} 1$, or the functional equivalent murine $\lg \mathrm{G} 2 \mathrm{c}$, can enhance the anti-tumor activity via antibodydependent cellular phagocytosis (ADCP) and antibody-dependent cellular cytotoxicity (ADCC) ${ }^{29,30}$. To determine whether this mechanism is also triggered by the upregulation of TAA antibodies in the carriers of this variant, we co-incubated necrotic exogenous antigen-expressing tumor cells of either LLC-M2e (Influenza virus M2 ectodomain, in three tandem repeats) or MC38-mOVA, with in vitro differentiated phagocytes in the presence of either anti-M2e IgG2c antibodies or OVA antiserum. We then calculated the efficiency of phagocytosis by flow cytometry and confocal fluorescence imaging. Indeed, anti-M2e IgG2c antibodies induced efficient phagocytosis of LLC-M2e cells by bone marrow derived macrophages 
(BMDMs) in a dose-dependent manner (Fig. 4A, Extended Data Fig. 7F). More importantly, OVA antiserum or further purified IgG from OVA-immunized mlgG2c-G400R mice with MC38-mOVA induction showed more potent effects on BMDM activation and phagocytosis towards antibody targeted tumor cells (Fig. 4B, Extended Data Fig. 7G). The enhanced ADCP activity of BMDMs largely relied on dominant TAA specific IgG2C antibodies in the serum on account of similar ADCP effects with purified IgG antibodies (Fig. 4C-E). Considering tumor antigen presentation by DCs is crucial to initiate T-cell-mediated protective immunity, we investigated the impact of mlgG2c-G400R on antibody-mediated tumor antigen uptake by FLT3L-DCs ${ }^{31,32}$. Similarly, FLT3L-DCs showed dose-dependent phagocytosis of antibody-coated tumor antigens examined by flow cytometry (Fig. 4F, Extended Data Fig. 7F, G) and confocal fluorescence microscopy (Fig. 4G). We found that purified IgG antibodies from OVA-immunized mlgG2c-G400R mice enhanced the antigen uptake activities of FLT3L-DCs (Fig. 4H, I), which in turn promoted OT-I CD8 ${ }^{+} \mathrm{T}$ cell activation and proliferation (Fig. $4 \mathrm{~J}, \mathrm{~K}$ ). Collectively, this variant strengthens anti-tumor effects in part by enhancing $A D C P$ and subsequent antigen presentation.

The enhanced anti-tumor immunity in mIgG2c-G400R mice prompted us to assess whether adoptive transfer of tumor-specific $B$ cells is a potential and feasible immunotherapy. Thus, we adoptively transferred MC38-primed B cells to B-cell-deficient mice ( $\mu \mathrm{MT})$ followed by MC38 tumor inoculation. Clearly, tumor primed B cells from mlgG2C-G400R mice conferred improved protection from tumor progression (Fig. 4L). We next assessed the roles of tumor-specific IgG on anti-tumor response by IgG reinfusion to $\mu \mathrm{MT}$ mice followed by MC38 tumor inoculation. $\mu \mathrm{MT}$ mice with IgG reinfusion showed significantly decreased MC38 tumor progression compared with untreated $\mu \mathrm{MT}$ mice (Fig. 4M). Furthermore, $\mu \mathrm{MT}$ mice showed much slower tumor growth after administration of IgG purified from MC38-inoculated mlgG2c-G400R mice (Fig. 4M). These results demonstrate the potential therapeutic effect of tumor-primed highly reactive B cells and the feasibility of BCR manipulation in future immunotherapy.

Although the impacts of B cells on tumor progression are still somewhat controversial based on tumor types and tumor stages in both human patients and murine disease model studies ${ }^{33,34}$, our study here uncovers the roles of $\mathrm{IgG} 1^{+} \mathrm{B}$ cells in antitumor immunity. A protective germline variant, hlgG1-G396R, which enhances $\lg \mathrm{G}^{+} \mathrm{B}$ cell activation and differentiation to plasma cells by potentiating the phosphorylation of IgG1 immunoglobulin tail tyrosine motif, fuels immune responses against tumors in at least four ways: (i) elevated and dominant tumor-specific IgG1 versus IgG3 production, which promotes avidity of macrophages, DCs and NK cells towards IgG1 bound on tumors 35,36 ; (ii) improved ADCP by macrophages and DCs; (iii) enhanced presentation of neoantigens to potentiate effector $C D 8^{+} T$ cell activation; and (iv) supports functional TLS formation, in which switched memory B cells are enriched in responders and synergize with killer T cells for effective tumor cell targeting. Concurrently, we expect that $B$ cells might also play additional roles. For example, activated tumor-infiltrating $B$ cells might secrete cytokines such as IFN- $y$ and tumor necrosis factor-alpha (TNF-a) to fine-tune the immune context in tumors ${ }^{37}$. In addition, hlgG1-G396R might alter the affinity of antibodies targeting neoantigens, and in turn block immune evasion of tumor cells. Future studies will be important to explore these phenomena 
and other potential mechanisms, and determine whether hlgG1-G396R has similar effects on survival and progression in other cancer types. Most importantly, the favorable antitumor outcomes after adoptive transfer of highly reactive $B$ cells in mice highlight the possibility of combined adoptive $T$ cell therapies with $B$ cells. Thus, our work suggests that $\lg G 1^{+} B$ cells might serve as tuners for orchestrating broad and robust immunosurveillance and provide a notion that harnessing human $\operatorname{lgG} 1^{+} B$ cells in the TME could be a powerful strategy for cancer immunotherapy.

\section{Materials And Methods}

\section{CRC Patients, healthy controls and ethics}

1006 colorectal cancer (CRC) patients with histological verification by Peking University People's Hospital and 583 healthy controls ( $\mathrm{HCs}$ ) were informed with for-in vitro study-purpose consents. After surgery, the CRC samples and tumor-adjacent tissues were immediately collected and separated as aliquots in cryogenic vials. All tissues were preserved at $-80^{\circ} \mathrm{C}$ before further treatments and assays. Basic features (gender, age, et al.), pathologic features (tumor location, tumor size, gross morphology, histological type, tumor differentiation, lymphovascular invasion, pathological TNM stage, clinical stage, et al.) and serum tumor markers (CEA, AFP, CA199, CA125, et al.) were documented in detail at Peking University People's Hospital. TNM staging was performed following the instructions of AJCC (7th edition). Overall survival (OS) was recorded and calculated from the time of surgery to the last follow-up or date of death due to CRC. Progression-free survival (PFS) was defined the time from the first time of disease progression or death from any cause. The re-usage and re-analyses of this CRC cohort for this study were approved by the Ethics Committee of Peking University People's Hospital (2020PHB046-02, 2020PHB047-01) and the Ethics Committee of Tsinghua University (20200073).

\section{TaqMan probe-based rs117518546 genotyping}

The genomic DNA of CRC patients and HCs were extracted from white blood cells. Genotyping of rs 117518546 was performed using the TaqMan Genotyping Master Mix (Thermo Fisher Scientific) and SNP Genotyping probes for rs 117518546 (Thermo Fisher Scientific) following the manufacturer's instructions and our published protcocols ${ }^{1}$.

\section{Reagents and antibodies}

Azoxymethane (AOM), ovalbumin (OVA) and bovine serum albumin (BSA) were purchased from SigmaAldrich. Dextran sodium sulfate (DSS) was purchased from MP Biomedicals. L-012 solution was purchased from WAKO chemicals. Mouse Fcy Receptor (CD16/CD32) blocking antibody, fixation and permeabilization solution and mouse IFN- $\gamma$ Cytometric Bead Array (CBA) were purchased from BD biosciences. FLT3L was purchased from PeproTech. M-CSF was purchased from BioLegend. Collagenase IV was purchased from VETEC. DNase I was purchased from ROCHE. L-Glutamine and penicillin/streptomycin were purchased from Gibco. Anti-mouse IgD, B220, CD38, CD56, CD20, CD138, GL-7, CD3, CD4, CD8, CD24, CD44, CD62L, CD11b, CD11c, H-2K'b bound to SIINFEKL, MHCII, CD45, NKp46, 
F4/80, IFN-ץ, PD-1, CD172, granzyme B, CD103 antibodies were purchased from BioLegend. Anti-mouse IgG2c, IgG1, IgG2b antibodies were purchased from Jackson ImmunoResearch Laboratory. TB Green ${ }^{\circledR}$ Premix Ex $\operatorname{Taq}^{\text {TM }}$ II was purchased from Takara. Protein A/G agarose pre-packed column, Fast Flow was purchased from Beyotime. Taq MasterMix was purchased from Tsingke. Hematoxylin staining solution was purchased from ZSGB Biotech. 10\% Formalin solution was purchased from Leagene Biotech. Tissue Grinder G50 was purchased from Coyote Bioscience. MEGAclear kit, MEGAshortscript ${ }^{\text {TM }}$ transcription kit $^{2}$ and CellTrace Violet (CTV) were purchased from Invitrogen. HiPure Total RNA Mini Kit was purchased from Magen. cDNA Synthesis Kit was purchased from Thermo Fisher. TH-Z93 adjuvant was a kind gift from professor Dr. Yonghui Zhang (Tsinghua University). Anti-m2e IgG2C antibody was a kind gift from professor Dr. Fan Wu (Fudan University).

\section{Cell culture and transfection}

MC38, LLC, B16F10 and HEK 293T cells were purchased from national Biomedical Cell Resource (BMCR, China). Generally, cells were cultured in complete DMEM medium containing $10 \%$ heat-inactivated fetal bovine serum, $2 \mathrm{mM} \mathrm{L-Glutamine} \mathrm{and} 100 \mathrm{U} / \mathrm{ml}$ penicillin/streptomycin. Retrovirus was constructed on the PHAGE backbone. MC38 and B16F10 cells were infected by retrovirus to stably express both fluorescent protein mCherry and exogenous membrane-bound OVA (MC38-mOVA, B16-mOVA). LLC cells were infected by retrovirus to stably express both fluorescent protein mCherry and exogenous membranebound m2e (influenza virus M2 extracellular domain) in three tandem repeats (LLC-m2e). Retrovirus infected tumor cells were sorted through Aria III basing on the expression levels of mCherry.

\section{Mice}

Rag1/- (B6.129S7-Rag $\left.1^{\text {tm } 1 M o m} / \mathrm{J}, 002216\right)$ and $\mu \mathrm{MT}$ (B6.129S2-lgh- $\left.6^{\operatorname{tm} 1 \mathrm{Cgn}} / \mathrm{J}, 002288\right)$ mice were obtained from professor Dr. Hai Qi (Tsinghua University). mlgG2c-tailless and mlgG2c-G400R genetically modified mice were generated by CRISPR/Cas 9 based gene manipulation in wild type C57BL/ 6 background, as described in detail below. Mice were maintained under specific-pathogen free conditions in the animal facility of Tsinghua University. Mice experiments were performed according to the governmental and institutional guidelines to guarantee animal welfare. All experimental studies were approved with an assurance identification number: 15-LWL2 and 19-LWL1 by the Institutional Animal Care and Use Committee (IACUC) of Tsinghua University.

\section{Construction of mlgG2C-G400R knock-in mouse and mlgG2C-tailless mouse}

mlgG2C-G400R and mlgG2C-tailless mice were constructed on the C57BL/ 6 mice background. Firstly, two optimal sgRNA target sequences were screen out from Crispr design website (https://zlab.bio/guidedesign-resources). These two sgRNA sequences are listed below: 5'-GCTCAGACCCTCCAAACTGT-3', 5'AGGATGGATGGGCTTCTGCA-3'. The homolog- directed repair (HDR) target gene template for mlgG2CG400R KI was of mouse IGHG2C sequence, containing two 800-bp homology arms flanking the mlgG2CG400R mutation site and mutated protospacer adjacent motif (PAM) sites. Cas9 mRNA and sgRNAs were transcribed into mRNA by MEGAshortscript ${ }^{\text {TM }}$ transcription kit (Invitrogen) in vitro, and the transcription 
products were further purified by MEGAclear kit (Invitrogen). Knock in mixture for mlgG2c-G400R mouse manipulation was prepared in total $10 \mu \mathrm{l}$ with final concentration sgRNA1 $(3 n g / \mu l)$, sgRNA2 $(3 n g / \mu l)$, Cas9 mRNA $(10 \mathrm{ng} / \mu \mathrm{l})$ and HDR template $(10 \mathrm{ng} / \mu \mathrm{l})$. Knock out mixture for mlgG2c-tailless mouse manipulation was prepared in total $10 \mu \mathrm{l}$ with final concentration sgRNA1 $(3 \mathrm{ng} / \mu \mathrm{l})$, sgRNA2 $(3 \mathrm{ng} / \mu \mathrm{l})$ and Cas 9 mRNA $(10 \mathrm{ng} / \mu \mathrm{l})$. The mixtures were delivered to mouse zygotes through microinjection. The generated mouse was verified through sequencing mouse PCR products, and mouse genotyping primers were described in detail below. The mlgG2c-G400R mouse and mlgG2c-tailless mouse were backcrossed to $\mathrm{C} 57 \mathrm{BL} / 6$ mouse for at least three generations.

\section{Mouse genotyping}

Genomic DNA was extracted from a section of the mouse tail. PCR amplification was performed using Taq MasterMix. PCR amplicons were sequenced. Genotyping primers for mlgG2c-tailless and mlgG2cG400R mice are listed following: forward, 5'-TCCTCCATTCCCTGAGCC-3'; reverse, 5'-

TGGTTCTTCTGGTCCGGAG-3'.

\section{Mice immunization and ELISA}

6-week-old gender-matched mlgG2c-tailless, WT and mlgG2c-G400R mice were injected with $100 \mu \mathrm{g}$ OVA (Sigma-Aldrich) in combination with $100 \mu \mathrm{g}$ TH-Z93 adjuvant through foot pad ${ }^{2}$. Serum samples were collected from tail vein on day 0 and weekly after immunization to quantify OVA-specific antibody titers. Sera samples were stored at $-80^{\circ} \mathrm{C}$ before further processing. To detect antibody levels, $2 \mu \mathrm{g} / \mathrm{ml}$ biotinconjugated goat anti-mouse IgG or $2 \mu \mathrm{g} / \mathrm{ml}$ goat anti-mouse IgA was coated on the ELISA plate overnight at $4^{\circ} \mathrm{C}$ to capture antibodies. ELISA plates were blocked with $5 \%$ skim milk in PBS at $37^{\circ} \mathrm{C}$ for 1 hour and then incubated with serum, colon explants culture medium or control culture medium for $2 \mathrm{~h}$. Peroxidaseconjugated immunoglobulin subclass (IgG, IgG1, IgG2b, IgG2c, IgG3, IgA)-specific antibodies were further used for final detection. To detect OVA-specific antibodies, $2 \mu \mathrm{g} / \mathrm{ml}$ OVA in PBS was coated on the ELISA plate.

\section{Tumor growth and treatment}

For MC38 cell inoculation-derived tumor model, $3 \times 10^{5}$ MC38 cells were re-suspended in $100 \mu$ sterile PBS and injected subcutaneously into the right flank of mice. For MC38-mOVA cell inoculation-derived tumor model, $2 \times 10^{6}$ MC38-mOVA cells were also re-suspended in sterile PBS and injected subcutaneously into the right flank of mice at week 6 after recall immunization with OVA. For B16-mOVA cell inoculationderived tumor model, $2 \times 10^{6}$ B16-mOVA cells in sterile PBS were injected intravenously into mice at week 6 after recall immunization with OVA. Tumor growth was measured every two days, and tumor size was calculated by length $(a)$ and width (b): tumor size $=0.52 \times a b^{2}$. Mice were sacrificed when tumor size reached to $2000 \mathrm{~mm}^{3}$.

\section{AOM/DSS induced colitis-associated carcinoma (CAC) model}


The AOM/DSS induced CAC model has been described previously ${ }^{3,4}$. Briefly, 10-week-old male WT or mlgG2C-G400R mice were injected intraperitoneally with a single dose of organotropic carcinogen AOM (10 mg/kg of body weight) on day 0 . Then mice were treated with $2.5 \%$ DSS (molecular weight 35-50 $\mathrm{kDa}$ ) in drinking water for 7 days in each turn (day 1-7, day 22-28, day 43-49), followed by recovery for 14 days with normal drinking water. Mice were monitored for weight loss twice a week. The serum samples were isolated at indicated time points in each figure legends. Mice were euthanized at week 16 after induction and the colons were isolated to assess the tumor generation.

\section{In vivo imaging of intestinal cancer-associated inflammation}

After isoflurane supported anesthesia, mice were intraperitoneally injected with $25 \mathrm{mg} / \mathrm{kg} \mathrm{L}-012$ solution (Wako Chemicals). Bioluminescent images of each mouse were obtained under isoflurane anesthesia using an IVIS Spectrum system (Perkin Elmer). For quantitative analyses, the Living Image software was utilized to calculate the intensity of bioluminescent signals at standardized regions of interest (ROIs) for each mouse.

\section{In vitro culture of colon explants}

The levels of IgG subclasses and IgA in colon explants were measured by ELISA. The method of colon explants in vitro culture was described previously ${ }^{5}$. Briefly, the fresh colon was collected from AOM-DSS induced mice and cut into pieces approximately $1 \mathrm{~cm}$ long. Then the colon tissue was washed vigorously in sterile PBS for three times, and in turn incubated in complete DMEM medium for 24 hours. To exclude unnecessary variables, colon pieces were also weighed before incubation, and colon pieces harboring equivalent wet weight were included into the in vitro culture procedure.

\section{Hematoxylin and Eosin (H\&E) staining and immunohistochemical (IHC) staining}

After euthanasia, colon specimens were immediately isolated from CAC-induced mice and fixed in 10\% formalin solution overnight, and colon specimens were subsequently embedded in paraffin. Standard hematoxylin-eosin (H\&E) staining was used to evaluate the pathological severity of colon tissues. For IHC analyses, slides were incubated with indicated primary antibodies in PBS containing 5\% BSA overnight at $4^{\circ} \mathrm{C}$. Then, streptavidin-HRP was added, and finally the slides were stained with both DAB staining kit and Hematoxylin staining solution (ZSGB-Bio). The slides were scanned by Axio Scan Z1 (Zeiss) and all the images were analyzed via Zen imaging software (Zeiss).

For IHC staining of human samples, all samples of tumor sections from 24 CRC patients were obtained with informed consent under a clinical protocol approved by the Ethics Committee of Peking University People's Hospital and the Ethics Committee of Tsinghua University.

\section{Tumor associated antigen (TAA) Microarray}

TAA microarrays were applied to measure TAA-specific antibodies by iGene Biotechnology. A HuProt TAA microarray containing 51 types of TAAs was utilized, which contains cell proliferation associated proteins 
(p53, c-myc, CDK2, BRCA1 and BRCA2, et al.), cancer antigens (CA-125, CA15-3, CA19-9, et al.), chemokine or cytokine and other tumor associated proteins (CEA, SOX2, NY-ESO-1, et al.).

TAA-specific $\lg M, \lg G 1, \lg G 2 b$ and $\lg G 2 c$ subclasses in the serum samples from CAC-induced mice and TAA-specific IgM, IgG1 and IgG3 subclasses in the plasma samples from CAC patients were measured. The serum samples and plasma samples were diluted 50 times and the secondary antibodies were diluted 1000 times.

\section{RNA extraction and RT-qPCR}

After isoflurane supported anesthesia, fresh colon specimens were immediately isolated and ground with Tissue Grinder G50 (Coyote Bioscience) to get colon tissue homogenate. HiPure Total RNA Mini Kit was utilized to extract total RNA following manufacturer's protocols. cDNA was then synthesized through reverse transcription according to manufacturer's protocol and examined by quantitative PCR analyses, and each group was detected in triplicate. All the qPCR primers are listed in detail as an online table.

\section{Adoptive transfer experiments}

Murine B cells from TDLNs and spleen were negatively purified utilizing AutoMacs Pro (Miltenyi Biotec). Splenic T cells were purified by Aria III. Purified B cells were then adoptively transferred into $\mu$ MT recipient mice $\left(1 \times 10^{7} B\right.$ cells per mouse). Purified $B$ cells and $T$ cells were mixed in a 1:1 ratio and then adoptively transferred into Rag $1 \%$ recipient mice. Twenty-four hours after adoptive transfer, recipient mice were inoculated with either MC38 cells or MC38-mOVA cells ( $2 \times 10^{6}$ cells per mouse) by subcutaneous injection. Tumor growth was monitored and recorded every two days. Mice were sacrificed when tumor size reached $2000 \mathrm{~mm}^{3}$. IgG was purified from the serum samples of either MC38-inoculated mice or OVA-immunized mice utilizing protein A/G agarose pre-packed column (Fast flow, Beyotime). The purified IgG was further dialyzed overnight in sterile PBS. For reinfusion experiments in vivo, $200 \mu \mathrm{g}$ purified IgG in $200 \mu \mathrm{l}$ sterile PBS was intravenously injected to $\mu \mathrm{MT}$ recipient mice on day 0, 5, 10 and15 after tumor cell inoculation ${ }^{6}$.

\section{Isolation of lamina propria lymphocytes (LPL) and tumor-infiltrating lymphocytes (TILs)}

For LPL preparation ${ }^{7}$, the colon was collected with fat, mesentery and intestinal contents carefully removed in cold HBSS buffer. The intestine was cut into $1 \mathrm{~cm}$ long pieces and incubated in epithelial strip buffer (HBSS, $5 \mathrm{mM}$ EDTA, $1 \mathrm{mM} \mathrm{DTT}, 5 \% \mathrm{FBS}, 15 \mathrm{mM}$ HEPES) at $37^{\circ} \mathrm{C}$ in a $200 \mathrm{rpm}$ shaking incubator for $30 \mathrm{~min}$. The colon pieces were washed twice and further incubated in enzyme solution $(2 \mathrm{mg} / \mathrm{ml}$ collagenase IV and $0.1 \mathrm{mg} / \mathrm{ml}$ DNase I) for 45 minutes at $37^{\circ} \mathrm{C}$. The LPL was released into the supernatant and collected into several vial of single cell suspension on ice for later usage. Subcutaneous tumor was isolated from tumor-bearing mouse and cut into $1 \mathrm{~mm}$ pieces. The tumor pieces were digested with enzyme solution at $37^{\circ} \mathrm{C}$ shaker for $1 \mathrm{~h}$. Digested products were filtered through $70 \mu \mathrm{m}$ cell strainers. Filtered cell suspensions were ready to be straining after washing twice by MACS buffer (PBS, 1\% FBS, 5 mM EDTA). 


\section{Flow cytometry}

Murine primary cells were separated from the spleen, bone marrow, lymph node, lamina propria, subcutaneous tumor tissues and maintained as single cell suspensions in MACS buffer. Single cell suspensions were pre-incubated with anti-mouse CD16/CD32 (BD bioscience) on ice for 30 min to block Fcy receptors, and then cells were stained with specific fluorescent dye-conjugated cell surface marker antibodies. Cells were also stained with either propidium iodide (PI) or DAPI to exclude the dead cells. After washing, cells were re-suspended in MACS buffer and analyzed by either LSRII or Symphony A5 (BD). Cell sorting was performed with the Aria III (BD). All data was further processed with FlowJo (V10) software. For IgG2 $\mathrm{c}^{+}$plasma cell staining, primary cells were first blocked and stained with fluorochromeconjugated rat anti-mouse B220 and CD138. After washing with MACS buffer, cells were fixed and permeabilized. Subsequently, cells were stained with Alexa Fluor 647-conjugated Fab fragment antimouse IgG2c, Fc specific (Jackson ImmunoResearch) on ice for 30 min before further FACS processing.

\section{Re-stimulation of tumor-primed B cells ${ }^{8}$}

B cells purified from TDLNs were activated in RPMI-1640 medium with $10 \mu \mathrm{g} / \mathrm{ml}$ LPS for 2 days. Activated $B$ cells were used for immune function analysis. Then the B cells were stimulated by irradiated MC38 cells. After 24 hours, the levels of IgG subclasses secretion in supernatants were detected by ELISA.

\section{Antibody-dependent cellular phagocytosis (ADCP) of tumor cells by FLT3L-DCs and BMDMs}

Primary cells were isolated from murine bone marrow and were then re-suspended into proper density. For FLT3L-DCs induction, primary cells were cultured in complete IMDM medium together with $100 \mathrm{ng} / \mathrm{ml}$ flt3l cytokine for 9-10 days. For BMDMs induction, primary cells were cultured in complete RPMI-1640 medium together with $20 \mathrm{ng} / \mathrm{ml} \mathrm{M-CSF}$ cytokine for 6 days ${ }^{9}$.

MC38-mOVA cells or LLC-m2e cells were prepared into necrotic cells (at least 3 frozen-thaw cycles) and co-cultured with either BMDMs or FLT3L-DCs as effector cells at a 1:1 ratio in the presence of specific antibody or antiserum. After co-culture, both flow cytometry and confocal fluorescence imaging methods were applied to distinguish tumor cells with phagocytic cells and calculated the efficiency of phagocytosis

After co-culture, tumor cells pulsed FLT3L-DCs (following methods mentioned above) were evaluated for their ability to prime OVA-specific OT-I CD8 ${ }^{+} T$ cells. Purified OT-I CD8 ${ }^{+} T$ Cells were stained by CTV following the instructions of user manual. DCs were sorted and co-cultured with OT-I CD ${ }^{+} \mathrm{T}$ cells at a 1:10 ratio. After either $48 \mathrm{~h}$ or $72 \mathrm{~h}$, cells were collected and stained with CD8 and PI. The levels of proliferation were indicated by CTV dilution by LSRII. DCs were able to cross-prime OT-I T cells to a greater extent mediating by antibody, which was clarified by increased percentages of proliferating OT-I $\mathrm{CD}^{+} \mathrm{T}$ cells. On day 3, Cytometric bead array (CBA) cytokine kit was applied to evaluate IFN- $y$ secretion by $C D 8^{+} T$ cells in supernatant following the manufacturer's instructions ${ }^{10}$. 
Olympus FLUOVIEW FV1000 confocal laser scanning microscope equipped with 4 lasers (405 nm, 473 $\mathrm{nm}, 557 \mathrm{~nm}$, and $635 \mathrm{~nm}$ ) for fluorescence excitation, 2 photomultiplier tubes (PMTs) for fluorescence detection, and a 10x objective lens was utilized. Images were processed and analyzed by Image Pro Plus software (Media Cybernetics).

\section{Statistical analysis}

Actuarial analyses on OS and PFS were performed basing on the detailed follow-up information. KaplanMeier analyses were carried out to build the survival curves, and log-rank tests were employed to assess the significance of the difference. R software v4.0.2 was used to analyze the probability of cumulative 5year and 100-month survival rates. Student's t-test or Wilcoxon signed-rank test was used to explore quantitative variables as appropriateness. Pearson's $\chi^{2}$ test or Fisher's exact test were used to explore categorical variables as appropriateness. Univariable and multivariable COX regression analyses were performed to identify prognostic factors. The Pearson $\chi^{2}$ test or binary logistic regression analysis were used for statistical analysis of allelic and genotypic differences between HCs and CRC patients. The measurements of statistical analysis were performed by using Prism 8.0.2 (GraphPad) and SPSS 23.0.

\section{Declarations}

Acknowledgments: We thank Dr. Yonghui Zhang, Dr. Michelle Meng Xu, Dr. Zhongjun Dong, Dr. Li Wu and Dr. Min Peng (all from Tsinghua University) for generously providing experimental materials. We thank Dr. Deming Wang (Medical College of Wisconsin), Dr. Chuan Wu (National Institutes of Health), Dr. Yan Li and Qing Sun (CAS Biobank) for helpful discussions and insightful comments. We thank Dr. Helen Pickersgill (life science editors) and Dr. Samina Shaheen for critical reading of this manuscript.

Funding: This work is supported by funds from National Natural Science Foundation of China (81825010, 81730043,81621002 and 81961130394 to W. Liu, 81972240 to Z. Shen).

Author contributions: W.L. and Z.S. conceived the project. B.Y., W.L., Z.S. and X.C. designed experiments. B.Y., X.W., L.D., W.S., Y.Z., X.S., X.Y. and J.G. performed experiments. Z.Z., C.Y., S.Z., Q.W. and L.Z. prepared CRC patient samples and collected clinical information. L.Z. and S.Q. analyzed scRNA-seq data. B.Y. and Z.Z. analyzed and interpreted the data. B.Y., X.C. and W.L. wrote the manuscript. W.L., X.C., Z.S., Z.Z., L.Z., X.W., L.D., L.Z. and H.Q. reviewed and revised the manuscript.

Declaration of interests: The authors declared no financial or commercial conflicts of interest.

Data and materials availability: Further information and requests for resources and reagents should be firstly directed to the lead contact, W.L. (liulab@tsinghua.edu.cn).

\section{References}


1 Waldman, A. D., Fritz, J. M. \& Lenardo, M. J. A guide to cancer immunotherapy: from T cell basic science to clinical practice. Nat Rev Immunol, doi:10.1038/s41577-020-0306-5 (2020).

2 Le, D. T. et al. PD-1 Blockade in Tumors with Mismatch-Repair Deficiency. N Engl J Med 372, 2509-2520, doi:10.1056/NEJMoa1500596 (2015).

3 Wei, S. C., Duffy, C. R. \& Allison, J. P. Fundamental Mechanisms of Immune Checkpoint Blockade Therapy. Cancer Discov 8, 1069-1086, doi:10.1158/2159-8290.cd-18-0367 (2018).

4 Topalian, S. L. et al. Safety, activity, and immune correlates of anti-PD-1 antibody in cancer. $N$ Engl J Med 366, 2443-2454, doi:10.1056/NEJMoa1200690 (2012).

$5 \quad$ Cabrita, R. et al. Tertiary lymphoid structures improve immunotherapy and survival in melanoma. Nature 577, 561-565, doi:10.1038/s41586-019-1914-8 (2020).

6 Helmink, B. A. et al. B cells and tertiary lymphoid structures promote immunotherapy response. Nature 577, 549-555, doi:10.1038/s41586-019-1922-8 (2020).

7 Petitprez, F. et al. B cells are associated with survival and immunotherapy response in sarcoma. Nature 577, 556-560, doi:10.1038/s41586-019-1906-8 (2020).

8 Tsou, P., Katayama, H., Ostrin, E. J. \& Hanash, S. M. The Emerging Role of B Cells in Tumor Immunity. Cancer Res 76, 5597-5601, doi:10.1158/0008-5472.CAN-16-0431 (2016).

9 Sharonov, G. V., Serebrovskaya, E. O., Yuzhakova, D. V., Britanova, O. V. \& Chudakov, D. M. B cells, plasma cells and antibody repertoires in the tumour microenvironment. Nat Rev Immuno/ 20, 294-307, doi:10.1038/s41577-019-0257-x (2020).

10 Chen, X. et al. An autoimmune disease variant of IgG1 modulates B cell activation and differentiation. Science 362, 700-705, doi:10.1126/science.aap9310 (2018).

11 Pincetic, A. et al. Type I and type II Fc receptors regulate innate and adaptive immunity. Nat Immuno/ 15, 707-716, doi:10.1038/ni.2939 (2014).

12 Okayasu, I., Ohkusa, T., Kajiura, K., Kanno, J. \& Sakamoto, S. Promotion of colorectal neoplasia in experimental murine ulcerative colitis. Gut 39, 87-92, doi:10.1136/gut.39.1.87 (1996).

13 Wirtz, S. et al. Chemically induced mouse models of acute and chronic intestinal inflammation. Nat Protoc 12, 1295-1309, doi:10.1038/nprot.2017.044 (2017).

14 Carmi, Y. et al. Allogeneic IgG combined with dendritic cell stimuli induce antitumour T-cell immunity. Nature 521, 99-104, doi:10.1038/nature14424 (2015).

15 Larsson, C. et al. Prognostic implications of the expression levels of different immunoglobulin heavy chain-encoding RNAs in early breast cancer. NPJ Breast Cancer 6, 28, doi:10.1038/s41523-020- 
0170-2 (2020).

16 Germano, G. et al. Inactivation of DNA repair triggers neoantigen generation and impairs tumour growth. Nature 552, 116-120, doi:10.1038/nature24673 (2017).

$17 \mathrm{Li}, \mathrm{Q}$. et al. Adoptive transfer of tumor reactive B cells confers host T-cell immunity and tumor regression. Clin Cancer Res 17, 4987-4995, doi:10.1158/1078-0432.CCR-11-0207 (2011).

18 Bolotin, D. A. et al. Antigen receptor repertoire profiling from RNA-seq data. Nat Biotechno/35, 908-911, doi:10.1038/nbt.3979 (2017).

$19 \mathrm{Hu}, \mathrm{X}$. et al. Landscape of B cell immunity and related immune evasion in human cancers. Nat Genet 51, 560-567, doi:10.1038/s41588-018-0339-x (2019).

20 Kaisho, T., Schwenk, F. \& Rajewsky, K. The roles of gamma 1 heavy chain membrane expression and cytoplasmic tail in IgG1 responses. Science 276, 412-415, doi:10.1126/science.276.5311.412 (1997).

21 Martin, S. W. \& Goodnow, C. C. Burst-enhancing role of the IgG membrane tail as a molecular determinant of memory. Nat Immuno/ 3, 182-188, doi:10.1038/ni752 (2002).

22 Xia, Y. et al. The Mevalonate Pathway Is a Druggable Target for Vaccine Adjuvant Discovery. Cell 175, 1059-1073 e1021, doi:10.1016/j.cell.2018.08.070 (2018).

23 Wei, Y. et al. Plasma Cell Polarization to the Immunoglobulin G Phenotype in Hepatocellular Carcinomas Involves Epigenetic Alterations and Promotes Hepatoma Progression in Mice.

Gastroenterology 156, 1890-1904 e1816, doi:10.1053/j.gastro.2019.01.250 (2019).

24 Ferris, S. T. et al. cDC1 prime and are licensed by CD4(+) T cells to induce anti-tumour immunity. Nature 584, 624-629, doi:10.1038/s41586-020-2611-3 (2020).

25 Sautes-Fridman, C., Petitprez, F., Calderaro, J. \& Fridman, W. H. Tertiary lymphoid structures in the era of cancer immunotherapy. Nat Rev Cancer 19, 307-325, doi:10.1038/s41568-019-0144-6 (2019).

26 Dieu-Nosjean, M. C., Goc, J., Giraldo, N. A., Sautès-Fridman, C. \& Fridman, W. H. Tertiary lymphoid structures in cancer and beyond. Trends Immuno/ 35, 571-580, doi:10.1016/j.it.2014.09.006 (2014).

27 Zhang, L. et al. Lineage tracking reveals dynamic relationships of T cells in colorectal cancer. Nature 564, 268-272, doi:10.1038/s41586-018-0694-x (2018).

28 Zhang, L. et al. Single-Cell Analyses Inform Mechanisms of Myeloid-Targeted Therapies in Colon Cancer. Cell 181, 442-459.e429, doi:10.1016/j.cell.2020.03.048 (2020).

29 DiLillo, D. J. \& Ravetch, J. V. Differential Fc-Receptor Engagement Drives an Anti-tumor Vaccinal Effect. Cell 161, 1035-1045, doi:10.1016/j.cell.2015.04.016 (2015). 
30 Nimmerjahn, F. \& Ravetch, J. V. Divergent immunoglobulin g subclass activity through selective Fc receptor binding. Science 310, 1510-1512, doi:10.1126/science.1118948 (2005).

31 Rafiq, K., Bergtold, A. \& Clynes, R. Immune complex-mediated antigen presentation induces tumor immunity. J Clin Invest 110, 71-79, doi:10.1172/jci15640 (2002).

32 Han, D. et al. Anti-tumour immunity controlled through mRNA m(6)A methylation and YTHDF1 in dendritic cells. Nature 566, 270-274, doi:10.1038/s41586-019-0916-x (2019).

33 Burger, J. A. \& Wiestner, A. Targeting B cell receptor signalling in cancer: preclinical and clinical advances. Nat Rev Cancer 18, 148-167, doi:10.1038/nrc.2017.121 (2018).

34 Wang, S. S. et al. Tumor-infiltrating B cells: their role and application in anti-tumor immunity in lung cancer. Cell Mol Immunol 16, 6-18, doi:10.1038/s41423-018-0027-x (2019).

35 Riechmann, L., Clark, M., Waldmann, H. \& Winter, G. Reshaping human antibodies for therapy. Nature 332, 323-327, doi:10.1038/332323a0 (1988).

36 Dahan, R. et al. FcgammaRs Modulate the Anti-tumor Activity of Antibodies Targeting the PD1/PD-L1 Axis. Cancer Cell 28, 285-295, doi:10.1016/j.ccell.2015.08.004 (2015).

37 Shen, P. \& Fillatreau, S. Antibody-independent functions of B cells: a focus on cytokines. Nat Rev Immuno/ 15, 441-451, doi:10.1038/nri3857 (2015).

\section{Method and material references}

1 Chen, X. et al. An autoimmune disease variant of IgG1 modulates $B$ cell activation and differentiation. Science 362, 700-705, doi:10.1126/science.aap9310 (2018).

2 Xia, Y. et al. The Mevalonate Pathway Is a Druggable Target for Vaccine Adjuvant Discovery. Cell 175, 1059-1073 e1021, doi:10.1016/j.cell.2018.08.070 (2018).

3 Okayasu, I., Ohkusa, T., Kajiura, K., Kanno, J. \& Sakamoto, S. Promotion of colorectal neoplasia in experimental murine ulcerative colitis. Gut 39, 87-92, doi:10.1136/gut.39.1.87 (1996).

$4 \quad$ Wirtz, S. et al. Chemically induced mouse models of acute and chronic intestinal inflammation. Nat Protoc 12, 1295-1309, doi:10.1038/nprot.2017.044 (2017).

5 Malik, A. et al. IL-33 regulates the IgA-microbiota axis to restrain IL-1 alpha-dependent colitis and tumorigenesis. J Clin Invest 126, 4469-4481, doi:10.1172/JCl88625 (2016).

$6 \mathrm{Gu}$, Y. et al. Tumor-educated B cells selectively promote breast cancer lymph node metastasis by HSPA4-targeting IgG. Nat Med 25, 312-322, doi:10.1038/s41591-018-0309-y (2019). 
7 Qiu, Z. \& Sheridan, B. S. Isolating Lymphocytes from the Mouse Small Intestinal Immune System. J Vis Exp, doi:10.3791/57281 (2018).

8 Li, Q. et al. Adoptive transfer of tumor reactive B cells confers host T-cell immunity and tumor regression. Clin Cancer Res 17, 4987-4995, doi:10.1158/1078-0432.CCR-11-0207 (2011).

9 Ferris, S. T. et al. CDC1 prime and are licensed by CD4(+) T cells to induce anti-tumour immunity. Nature 584, 624-629, doi:10.1038/s41586-020-2611-3 (2020).

10 Han, D. et al. Anti-tumour immunity controlled through mRNA m(6)A methylation and YTHDF1 in dendritic cells. Nature 566, 270-274, doi:10.1038/s41586-019-0916-x (2019).

Figures 


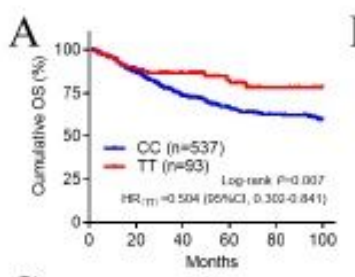

C Univariable risk factors
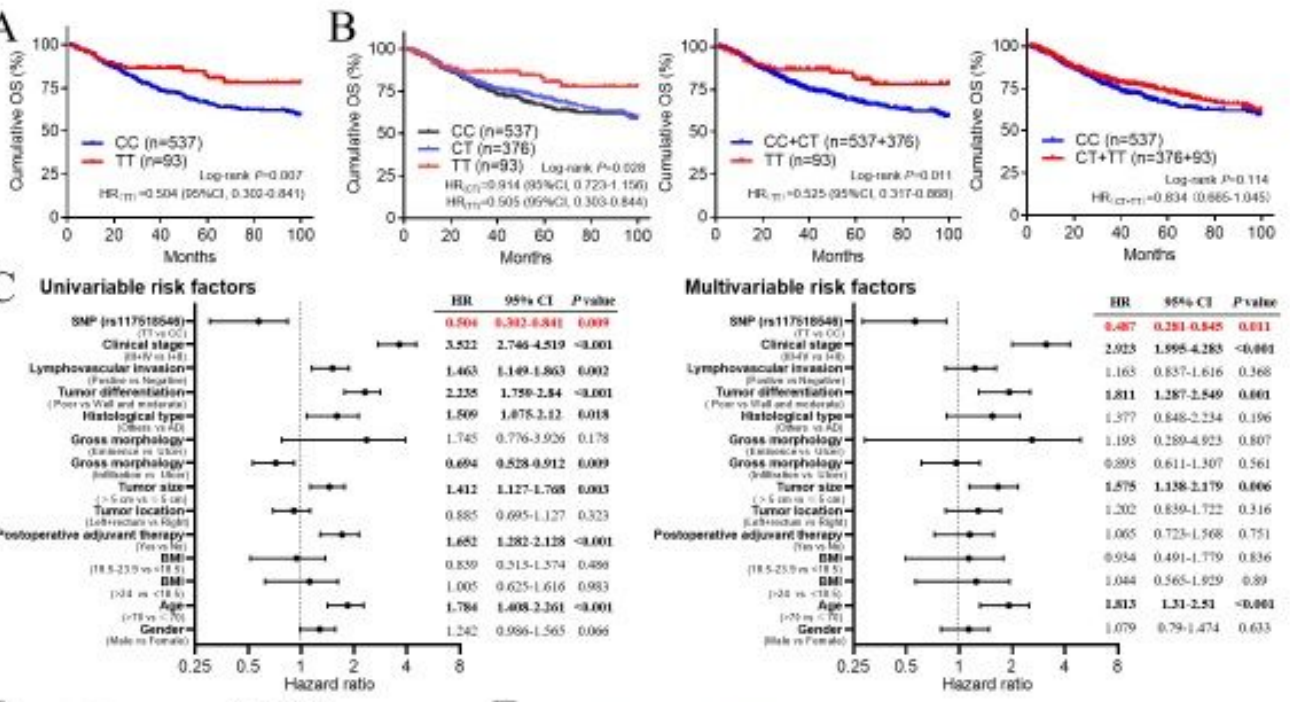

D

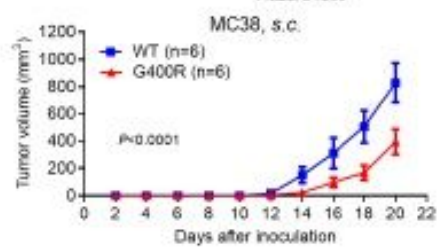

F

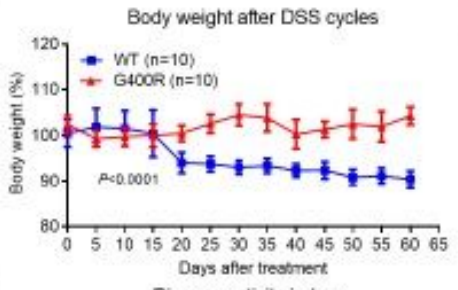

G

Disease activity index

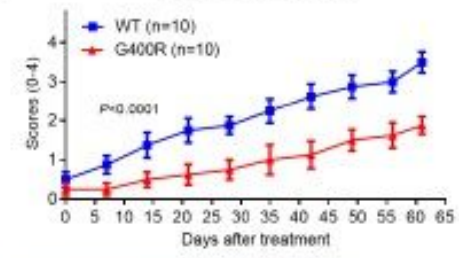

I

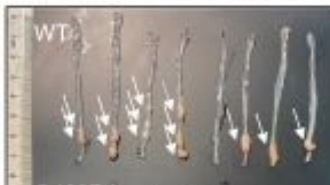

- G400R
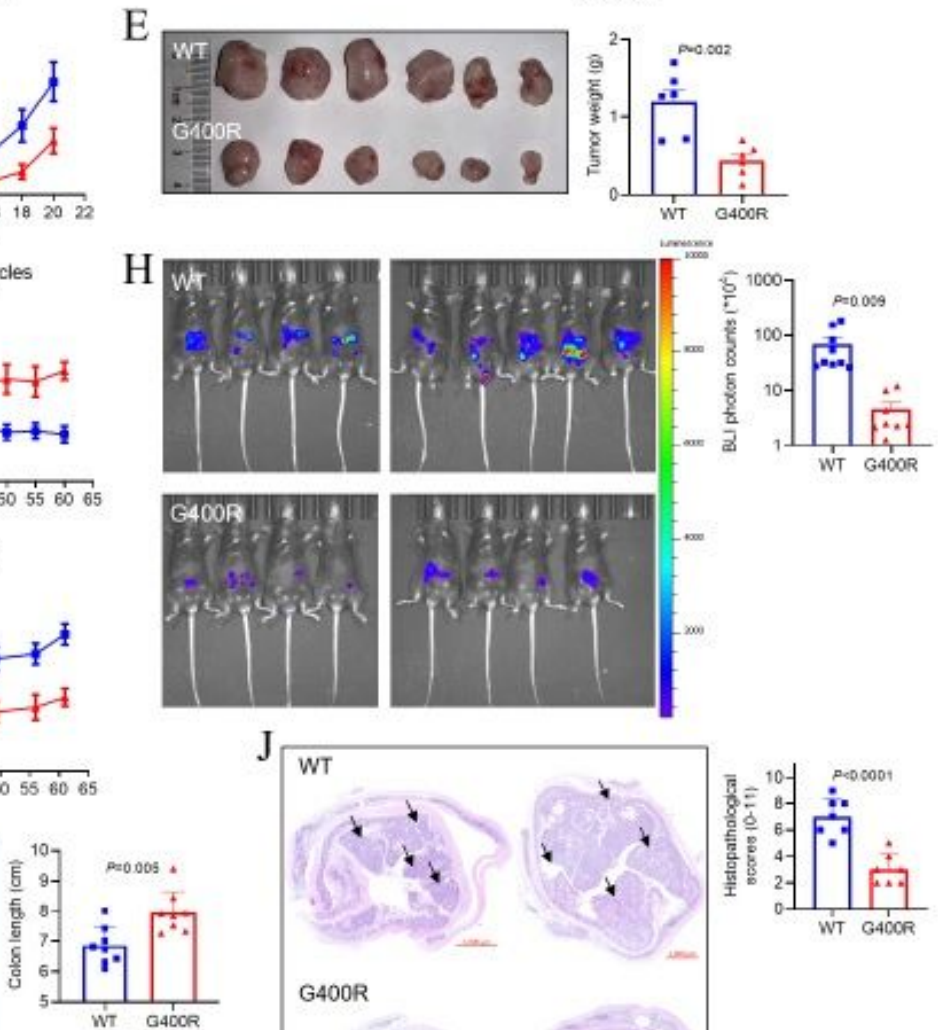

G400R
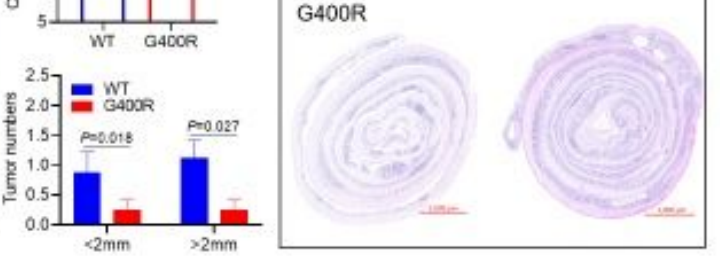

\section{Figure 1}

The hlgG1-G396R variant protects against colorectal tumorigenesis a $1 \mathrm{nd}$ progression(A) Survival analyses of $1006 \mathrm{CRC}$ patients with available follow-up information stratified by different genotypes. The proportions of 5-year cumulative OS were $66.41 \%(95 \% \mathrm{Cl}, 62.12 \%-71 \%)$ for WT (denoted as CC) patients, $77.93 \%$ (66.14\%-76.06\%) for hlgG1-G396R heterozygous (denoted as CT) patients and $80.77 \%$ (72.09\%-90.49\%) for hlgG1-G396R homozygous (denoted as TT) patients. (B) Survival curves for 1006 
CRC patients, classified by additive model (left), recessive model (middle) and dominant model (right), to assess the effects of hlgG1-G396R on OS. (C) Forest plots showing hazard ratios by univariable COX regression and multivariable COX regression analysis for correlation with the OS of CRC patients. MC38 tumor growth (D) and weight at the time of sacrifice (E) of WT and mlgG2c-G400R mice. Body weights (F) and disease activity index (G) of AOM-DSS induced CAC model for both WT and mlgG2c-G400R mice. $(\mathrm{H})$ Bioluminescent images with injection of L-012 solution at week 16 after the third cycle of DSS treatment. (I) Representative image and quantification of colon tumor numbers in mice of indicated genotypes at week 16 after treatment with AOM-DSS. Tumors are indicated by white arrows. (J) Representative photographs of H\&E-stained colon cross-sections and histopathological scores after termination of the experiment. The black arrows indicate the tumors in the colon. Scale bar, 1000 $\mu \mathrm{m}$. One of three representative experiments is shown (D-I). Statistical significance was determined using a Log-rank test (A, B), unpaired two-tailed t-test $(E, H, I, J)$ and two-way ANOVA (D, F, G). HR, hazard ratio. $\mathrm{Cl}$, confidence interval. Mean \pm SEM. 
A

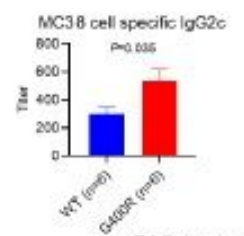

D

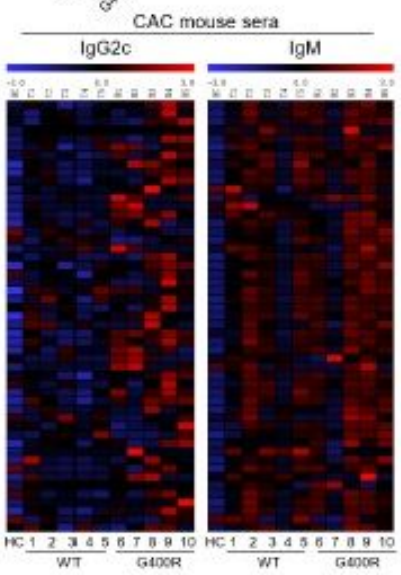

F
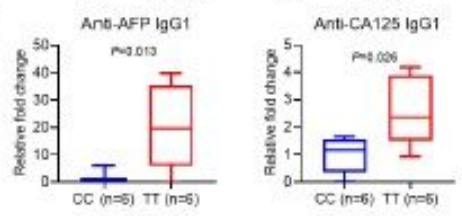

$\mathrm{H}$

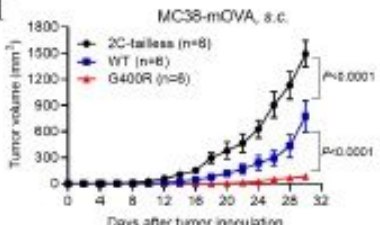

$\mathbf{J}$
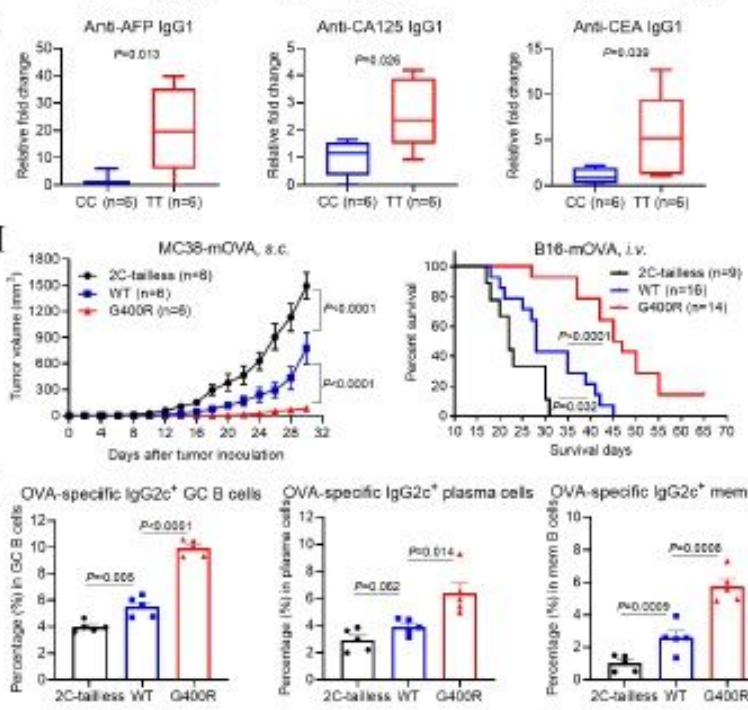

E

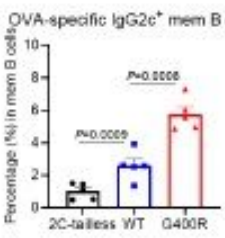

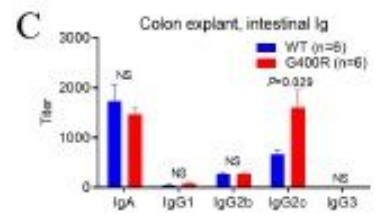

CRC patient plasma
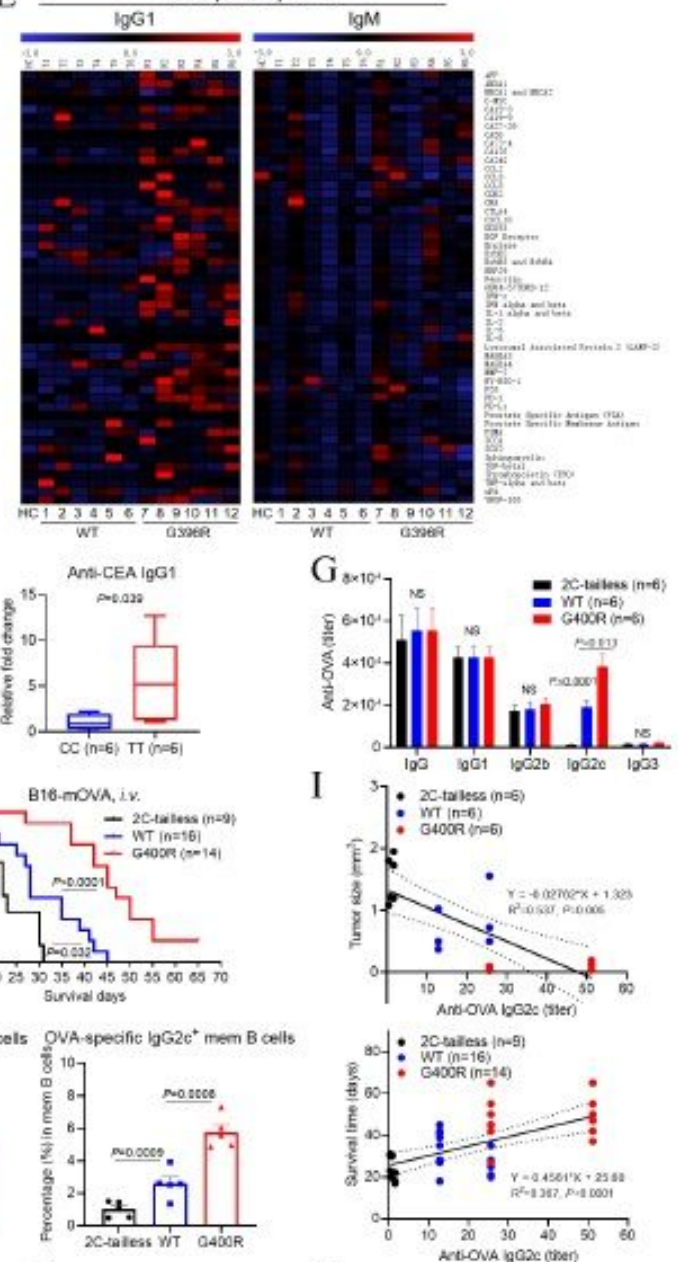

K ovh-specific lgG2et plasma
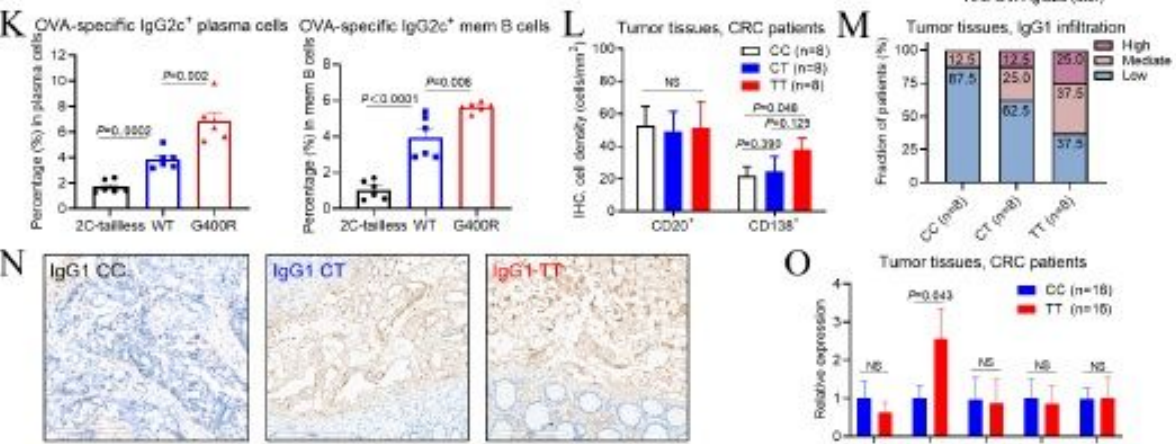

$\mathrm{O}$

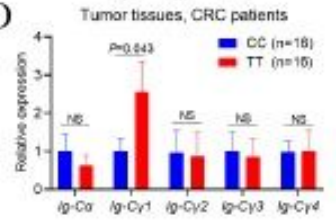

\section{Figure 2}

The hlgG1-G396R variant promotes tumor-specific 21 antibody production and plasma cell differentiation (A) Detection of MC38-specific IgG2c by ELISA in serum on day 20 post tumor inoculation. (B) Detection of IgG subclasses secretion in supernatants of in-vitro activated MC38 TDLN B cells.

(C) Quantification of IgG subclasses and IgA in supernatants of colon explants isolated from CAC-bearing mice through ELISA. (D) HuProt microarray containing recombinant TAAs and autoantigens to detect the 
antibodies specific for tumor-associated antigens in the serum samples of CAC-induced mice. (E) Heatmap showing the detection of TAAs and autoantigens specific IgG1 and IgM in the plasma samples of 12 colorectal cancer patients and 1 healthy donor. T1-T6 are equal to WT homozygotes. R1-R6 means hlgG1-G396R homozygotes. (F) Relative levels of anti-AFP IgG1, anti-CA125 IgG1 and anti-CEA IgG1 in the plasma samples from CRC patients, detected by TAA microarray assays. (G) Detection of anti-OVA antibodies in serum from mlgG2c-tailless, WT and mlgG2c-G400R mice at week 2 after recall immunization with OVA. $(\mathrm{H})$ Tumor growth of MC38-mOVA (left) or survival curves after intravenous injection of B16-mOVA tumor cells (right) at week 6 after recall immunization with OVA. (I) Linear regression analyses between the titer of OVA-specific lgG2c and tumor size (upper) or survival day (lower), as indicated by solid lines. Dotted lines indicate $95 \% \mathrm{Cl}$. (J-K) Flow cytometry analyses to assess the relative percentages of OVA-specific IgG2C+ GC B cells, memory B cells, and plasma cells in the spleen $(\mathrm{J})$ and bone marrow (K) from mlgG2c-tailless, WT and mlgG2c-G400R mice week 2 after recall immunization. ( $\mathrm{L})$ The absolute numbers of $\mathrm{B}$ cells (CD20+) and plasma cells (CD138+) in tumor tissues from CRC patients detected by IHC staining. Each genotype contains 8 individuals. (M) Elevated proportions of hlgG1-G396R homozygous CRC patients have higher levels of IgG1 infiltration. $(\mathrm{N})$ Representative microphotographs of IgG1 staining from IHC samples. Scale bar, $200 \mu \mathrm{m}$. (0) The transcriptional levels of germline immunoglobulin a-chain constant region $(\mathrm{Ca})$ and $\gamma$-chain constant region (CY1), CY2, CY3 and C 4 in the tumor tissues of CRC patients, measured by RT-qPCR analyses. One of three representative experiments is shown (A-C, G-K). Statistical significance was determined using an unpaired two-tailed t-test (A-C, F, G, J, K), two-way ANOVA $(\mathrm{H})$, Log-rank test $(\mathrm{H})$ and unpaired one-tailed ttest $(L, O)$. Mean \pm SEM. NS, not significant. 


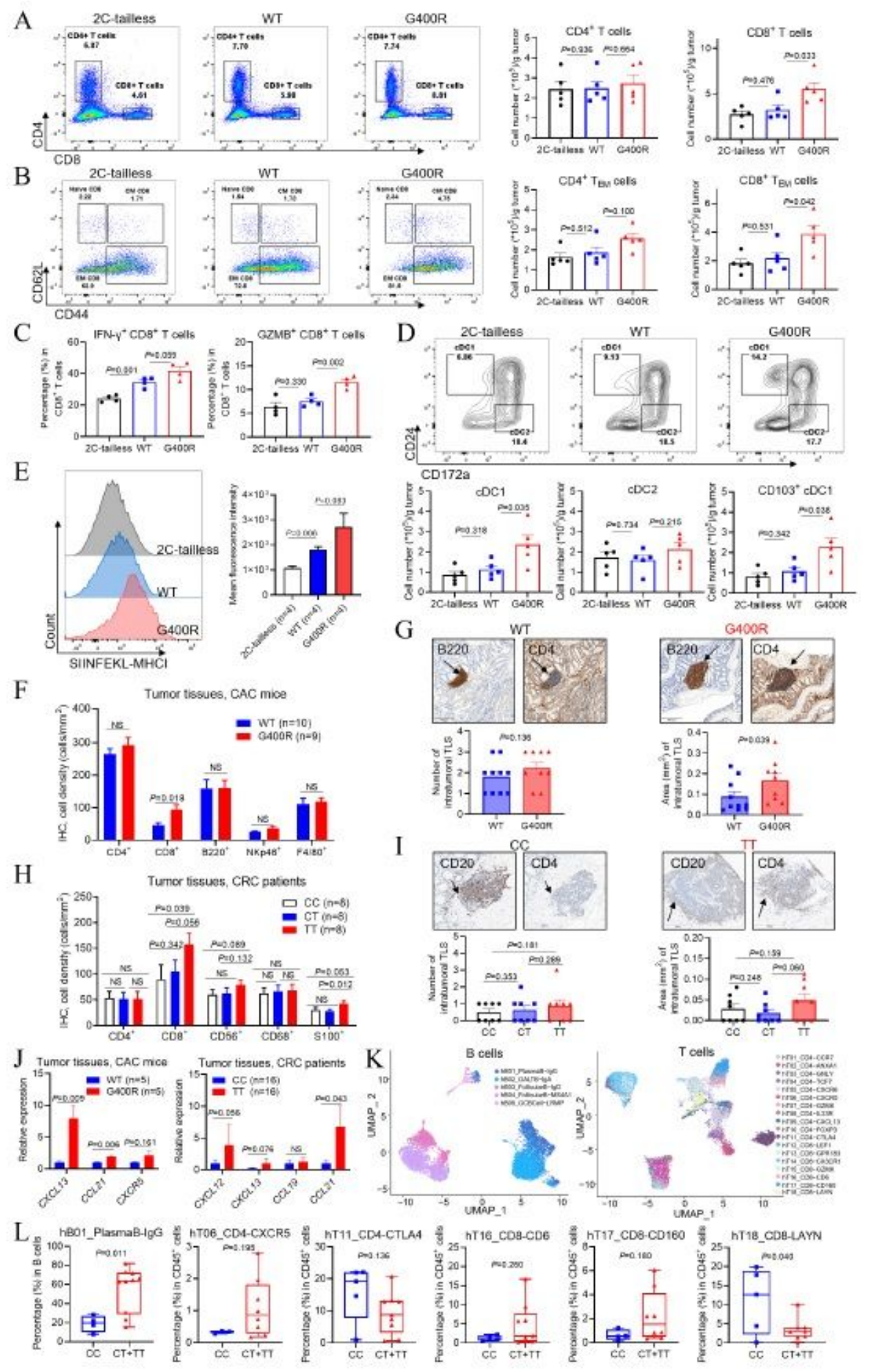

\section{Figure 3}

Elevated numbers of tumor infiltrating effector T cells 50 and DCs, and particularly increased formation of TLS in the TME of the hlgG1-G396R variant (A) Quantification of tumor-infiltrating CD4+ T cells and CD8+ T cells in the tumor tissues at day 20 post MC38-mOVA tumor cell inoculation by flow cytometry. Pre-gated on CD45+ cells. (B) Quantification of tumor-infiltrating naïve, central memory, effector memory CD4+ $T$ cell and CD8+ $T$ cell in the tumor tissues at day 20 post MC38-mOVA tumor cell inoculation by 
flow cytometry. (C) Quantification of IFN-y secreting and granzyme B (GZMB) secreting CD8+ T cells. Pregated on CD45+ CD8+ T cells. (D) Quantification of DC subtypes infiltrated in the tumor tissues.

(E) Expression levels of the SIINFEKL-MHC I complex on CD103+ CDC1 cells from (D). (F) The cell density (in mm2) of T helper cells (CD4+), T cytotoxic cells (CD8+), B cells (B220+), NK cells (NKp46+) and macrophages $(\mathrm{F} 4 / 80+)$ in colon specimens from CAC-induced WT and mlgG2C-G400R mice measured by IHC staining. (G) Microphotographs of representative TLS located in the tumor section of CAC-induced mice. IHC staining of the TLS showing the numbers and relative areas of TLSs within the tumor regions of the colon from CAC-induced mice. Black arrows indicate TLSs. Scale bar, $200 \mu \mathrm{m}$. (H) Quantification of cell density (in mm2) of tumor-infiltrating immune cells in tumor sections from hlgG1-G396R homozygous, heterozygous and WT CRC patients by IHC staining, including T helper cells (CD4+), T cytotoxic cells (CD8+), natural killer cells (CD56+), macrophages (CD68+) and DCs (S100+). (I) Representative IHC staining of TLSs within the tumor specimens from CRC patients, as indicated by black arrows. The numbers of TLSs per $\mathrm{mm} 2$ and relative TLS area for each genotype are shown. Scale bar, $200 \mu \mathrm{m}$. (J) The expression levels of several chemokine genes, associated with TLS formation, in the colon tumor tissues from CAC-induced mice (left) and CRC patients (right) by RT-qPCR assays. (K) Representative UMAP plot showing the clusters of B cells (left) and T cells (right) from 18 CRC patients analyzed by scRNA-seq. $(J)$ The proportions of tumor-infiltrating IgG+ plasma cells within total B cells, CXCR5+ T follicular helper cells, CTLA4+ T regulatory cells, CD6+ tumorresident memory T cells, CD160+ intraepithelial CD8+ T cells and LAYN+ exhausted T cells within total CD45+ cells from the scRNA-seq results. One of three representative experiments is shown (AE). Statistical significance was determined using an unpaired two-tailed t-test $(A-F, H, L)$ and unpaired one-tailed t-test $(G, J, I)$. Mean \pm SEM. NS, not significant. 
A Ant-M2e $\lg \mathrm{G} 2 \mathrm{c}$

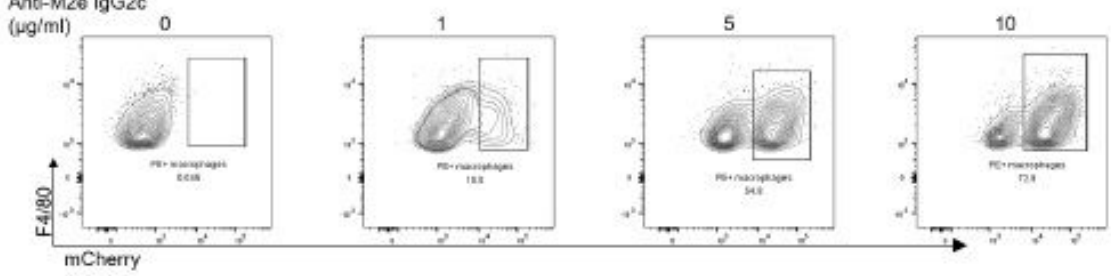

B

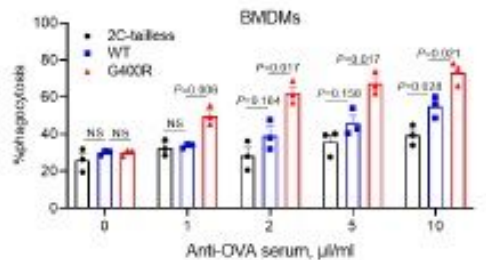

D

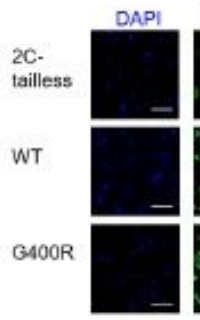

F

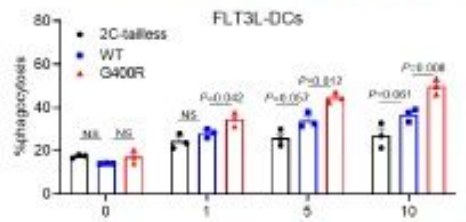

$\mathrm{H}$

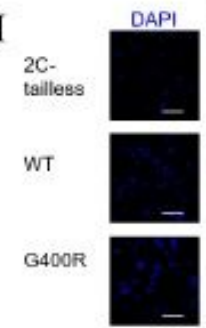

J
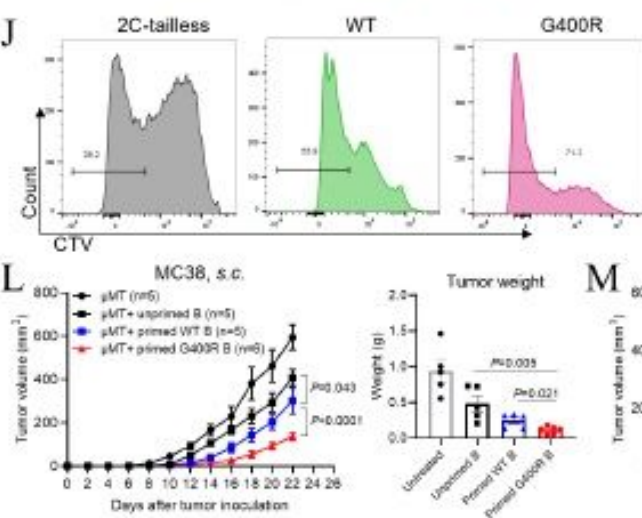

Devs ator tumer inositston
C
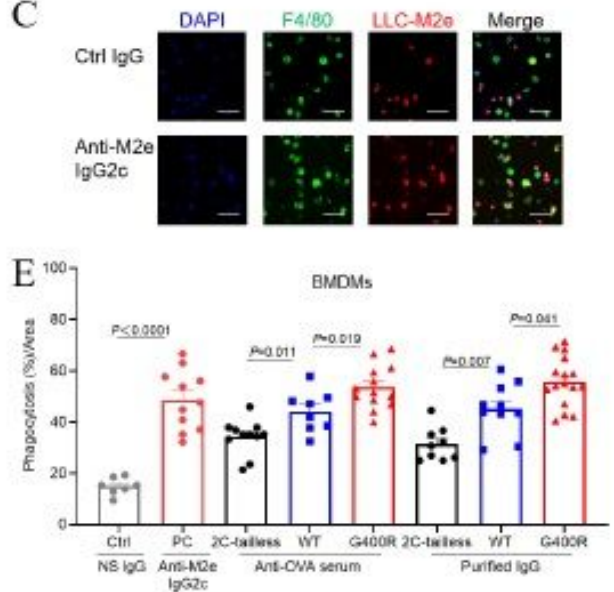

G
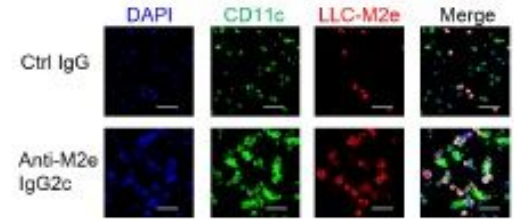

FLT3L-DCS
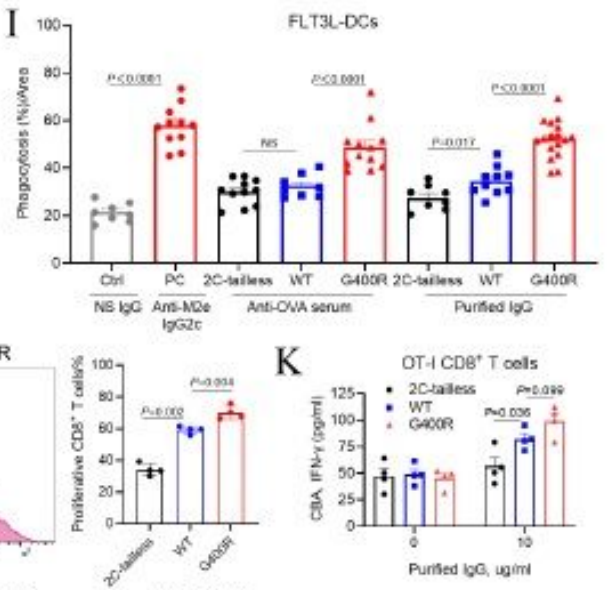

K OT- CDE' Tools

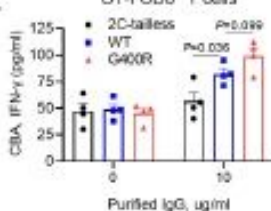

MC $38,5.0$

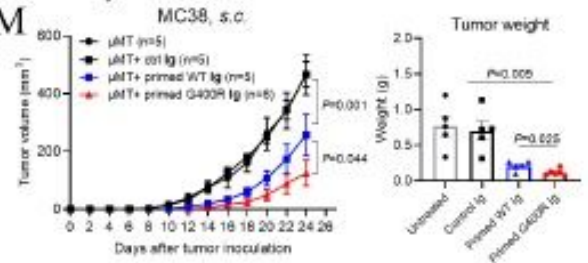

Figure 4

Enhanced ADCP activity, CD8+ T cells priming and tumor resistance 80 are mediated by the tumorspecific antibodies and tumor-primed $B$ cells from the mlgG2c-G400R variant (A) Representative flow cytometry plots to compare the ADCP activities of BMDMs with the presence of anti-M2e lgG2c antibody in different concentrations $(0,1,5,10 \mu \mathrm{g} / \mathrm{ml})$. ADCP activities of BMDMs were assessed by the proportions of mCherry+ and F4/80+ BMDMs. (B) Comparison of ADCP activities of necrotic tumor cells 
by BMDMs using serum samples from OVA-immunized mlgG2c-tailless, WT and mlgG2c-G400R mice, detected by flow cytometry. (C-D) Assessment of the ADCP efficiency of BMDMs by fluorescence staining. Representative images showing the cell mixtures after co-culture. Nuclei marked by DAPI staining (blue), macrophages labeled by F4/80-FITC (green), and engulfed tumor cells by mCherry (red). Scale bar, 50 $\mu \mathrm{m}$. (E) Percentage phagocytosis of tumor cells by BMDMs were analyzed based on the results from (C, D). (F) ADCP activities of necrotic tumor cells by FLT3L-DCs examined by flow cytometry. (G-H) Representative confocal fluorescence images showing the process of phagocytosis mediated by FLT3LDCs in the presence of tumor cell specific antibody. Nuclei marked by DAPI staining (blue), DCs were stained with CD11C-FITC (green) and tumor cells labeled by mCherry (red). Scale bar, $50 \mu \mathrm{m}$. (I) The percentages of mCherry+ DCs were recorded and used to calculate the levels of phagocytosis. (J) Quantification of CTV-labeled OT-I CD8+ T cells which had been co-cultured with antibodycoated tumor cell primed DCs. CD8+ T cells with CTV-low indicated proliferating OT-I CD8+ T cells. (K) Production of IFN- $\gamma$ by OT-I CD8+ T cells after co-culturing with tumor-primed FLT3L-DCs, detected by IFN- $\gamma$ cytometric bead array (CBA). (L) B cells purified from untreated mice, MC38 tumor bearing WT or mlgG2c-G400R mice were adoptively transferred into $\mu \mathrm{MT}$ mice followed by MC38 tumor inoculation. (M) IgG purified from the serum samples of untreated mice, MC38 tumor-inoculating WT or mlgG2c-G400R mice were intravenously reinfused into $\mu \mathrm{MT}$ mice followed by MC38 tumor cell inoculation. Tumor growth was recorded and tumor weight is shown. One of three representative experiments is shown. Statistical significance was determined using an unpaired two-tailed t-test (B, E, F, I, J, K, L, M) and two-way ANOVA $(L, M)$. Mean \pm SEM. NS, not significant.

\section{Supplementary Files}

This is a list of supplementary files associated with this preprint. Click to download.

- ExtendedDataFigure1.pdf

- ExtendedDataFigure2.pdf

- ExtendedDataFigure3.pdf

- ExtendedDataFigure4.pdf

- ExtendedDataFigure5.pdf

- ExtendedDataFigure6.pdf

- ExtendedDataFigure7.pdf

- ExtendedDataFigure8.pdf

- ExtendedDataFigure9.pdf

- ExtendedDataTables.docx

- flatnreditorialpolicychecklist0124.pdf

- flatnrreportingsummary0124.pdf 